\title{
Ragionevolezza della fede. Rapporto tra fede e ragione in Tommaso d'Aquino
}

\section{(Rationality of faith. Relationship between faith and reason in Thomas Aquinas)}

\section{MICHAŁ OLEKSOWICZ}

Pontifical Lateran University, Rome

oleksowicz.michal@gmail.com

Sommario. La razionalità della fede cristiana è una tematica discussa per secoli. Il rapporto tra fede e ragione è una realtà intrinseca del sapere cristiano. Ma, d'altra parte, dall'inizio il cristianesimo è stato una forma di religione basata sull'assenso di fede e non su una giustificazione umana. Qui nasce il cuore del problema nel rapporto tra fede cristiana e ragione, perché l'oggetto della fede non è evidente. Invece, in termini generali la conoscenza razionale si basa sull'evidenza e i principi della logica. Ma l'atto di fede, come dimostra San Tommaso, è qualcosa di più rispetto alla razionalità: "atto dell'intelletto dà l'assenso alla verità divina sotto la spinta della volontà mossa da Dio per grazia". S'intende dimostrare, sulla base del pensiero di San Tommaso, che la sua posizione possa considerarsi come un tentativo di realizzare un equilibrio riguardando il rapporto tra fede e ragione. Da una parte la fede non cade nell'irrazionalità del fideismo. D'altro canto, la fede nella concezione tommasiana non è stata ridotta a una forma di razionalismo. Un tentativo di rileggere il pensiero tommasiano può essere molto fecondo per promuovere un dialogo fra fede e ragione.

Parole chiave: teologia naturale; filosofia della religione; Aquinate; preambula fidei.

Abstract. The rationality of the Christian faith has been an issue debated for centuries. The relationship between faith and reason is an intrinsic reality of Christian thought. But on the other hand from the beginning the Christianity it was a form of religion 
based on the assent of faith and not on a human justification. Here comes the heart of the problem in the relationship between Christian faith and reason, because the object of faith is not obvious. Instead, in general terms the rational knowledge is based on evidence and the principles of logic. But the act of faith, as evidenced by St. Thomas, is something more than the simple rationality: "act of the intellect gives assent to the divine truth under the influence of the will moved by God's grace". It is intended to demonstrate, on the basis of the thought of St. Thomas, that his position clearly can be considered as an attempt to achieve a balance covering the relationship between faith and reason. On the one hand, faith does not fall into irrationality of fideism. On the other hand, the faith in the thomistic conception has not been reduced to a form of rationalism. An attempt to read again the thomistic thought can be very fruitful to promote a dialogue between faith and reason.

Keywords: natural theology; philosophy of religion; Aquinas; preambles of faith.

\section{Introduzione}

La domanda filosofica sull'esistenza di Dio emerge dall'inizio della storia dell'uomo. Nelle diverse culture, nei diversi momenti storici, si parla di Dio o del "divino". Possiamo dire che il problema di Dio sia un problema esclusivamente e propriamente umano. Esclusivamente in quanto soltanto gli uomini affermano o negano la sua esistenza; e propriamente perché in qualunque esperienza religiosa il "divino" è sempre presente come qualche Essere superiore e di ordine divino (Pangallo 2004, 9-21). Osserviamo da una parte, che nella cultura contemporanea, senza dubbio, esiste una peculiare attività della ragione umana, ma dall'altra parte, la ragione, invece di esprimere al meglio la tensione verso la verità, diventa pian piano incapace di sollevare lo sguardo verso l'alto. Come giustamente afferma Giovanni Paolo II "di recente, poi, hanno assunto rilievo diverse dottrine che tendono a svalutare perfino quelle verità che l'uomo era certo di aver raggiunto. La legittima pluralità di posizioni ha ceduto il posto ad un indifferenziato pluralismo, fondato sull'assunto che tutte le posizioni si equivalgono: è questo uno dei sintomi più diffusi della sfiducia nella verità che è dato verificare nel contesto contemporaneo" (1998, n. 5). In altre parole, si può esprimere questa "sfiducia nella verità" come "crisi del senso" che spesso deriva dalla frammentarietà del sapere come il risultato dei molti punti di vista, spesso di carattere scientifico-particolare (Fisichella 1997, 15-30). Di conseguenza 
"proprio questo rende difficile e spesso vana la ricerca di un senso" (Giovanni Paolo II 1998, n. 81). In questo contesto culturale sarebbe molto importante fare un tentativo così da dare uno sguardo sul rapporto fra ragione e fede nella dottrina cristiana, ma l'autore si limita a dimostrarlo secondo la speculazione filosofico-teologica di Tommaso d'Aquino: "Riaffermando la verità della fede, possiamo ridare all'uomo del nostro tempo genuina fiducia nelle sue capacità conoscitive e offrire alla filosofia una provocazione perché possa recuperare e sviluppare la sua piena dignità" (Giovanni Paolo II 1998, n. 6).

Il pontefice Benedetto XVI molte volte durante il suo pontificato ha insistito sul rifiuto del fideismo in ogni sua forma, perché voleva dimostrare che le ragioni per credere sono valide, ma non solo se comprese come "imperativi categorici" meramente formali o come un "dover credere" infondato, ma come ragioni, cioè come argomenti capaci di interpellare la razionalità umana. Ma questa razionalità umana, d'altra parte, non sempre è stata intesa nel senso riduttivo. Perché la razionalità intesa correttamente, non significa un arbitrario razionalismo che riduce la ragione solo alla funzione dimostrativa o alla verifica empirica, ma sia costituita anche di esperienza vitale, di ruolo della testimonianza, di percezione dei valori (Livi 2008, 325). S'intende dimostrare, sulla base del pensiero di San Tommaso, che la sua posizione possa considerarsi come un tentativo di realizzare un equilibrio riguardo al rapporto tra fede e ragione. Da una parte, la fede non cade nell'irrazionalità del fideismo. D’altro canto, la fede nella concezione tommasiana non è stata ridotta a una forma di razionalismo. Un tentativo di rileggere il pensiero tommasiano può essere molto fecondo per promuovere un dialogo fra fede e ragione.

\section{La prospettiva storica}

All'inizio dovrebbe essere chiarito che il rapporto tra fede e ragione è una realtà intrinseca del sapere cristiano. Si possono prendere due fondamentali testi dal Nuovo Testamento. Il primo è il brano dagli Atti degli Apostoli $(17,15-34)$. Il contesto è quello della predica di San Paolo all'Areopago di Atene. Bisogna leggere questo testo, inserendosi nella prospettiva dei 
rapporti tra predicazione del cristianesimo e strumenti dell'intelligenza umana, come il primo incontro tra cristianesimo e filosofia. Il contenuto importante da ravvisare è l'accettabilità della fede da parte dei filosofi e l'accettazione delle tesi dei filosofi da parte dei cristiani. Si può dire che il cristianesimo ha "i suoi precursori e la sua preparazione nella razionalità filosofica, non nelle religioni” (Ratzinger 2000, 45). Un secondo testo è il prologo del Vangelo di Giovanni $(1,1-18)$ nel quale è scritta la dossologia di Gesù-Logos. Il Prologo celebra gli attributi del Logos e si parla di un principio divino "per mezzo" del quale sono state create tutte le cose. Il testo racconta che "Il Verbo si fece carne", cioè Dio entra in questo mondo. Siamo davanti a un caso palese della filosofia utilizzata per dire qualcosa che va oltre la filosofia: "la rivelazione immette nella storia un punto di riferimento da cui l'uomo non può prescindere” (Giovanni Paolo II 1998, 14). Quindi, il cristianesimo inizia ad affermarsi, passando dal doversi difendere al momento della sua diffusione. Fin dal suo inizio era l'esigenza presso i cristiani di trovare una via razionale per accostarsi al cristianesimo, intesa, appunto, come religione del Logos. Questo era fondamentale per mostrare ai pagani come il cristianesimo non è superstizione, perché il Logos è l'ordine dell'universo (Fisichella 1997, 31-38).

Nell'Impero Romano la disponibilità dei Romani all'accogliere le religioni dei conquistati era un favorire la loro integrazione. Questo era un sincretismo religioso (la realizzazione in campo religioso così detta "pax romana”), perchè per i Romani la religione non era una cosa molto importante. Ma perché il cristianesimo è stato perseguitato?

Ed è proprio questo che nella vasta tolleranza dei politeismi doveva necessariamente apparire come intollerabile, addirittura come nemico della religione, come “ateismo". Non si fondava sulla relatività e sulla convertibilità delle immagini, disturbava perciò soprattutto l'utilità delle religioni, e metteva così in pericolo i fondamenti dello Stato, nel quale non voleva essere una religione tra le altre, ma la vittoria dell'intelligenza sul mondo delle religioni (Ratzinger 2000, 46).

Fin dall'inizio il cristianesimo era una forma di religione basata sull'assenso di fede e non su una giustificazione umana. È evidente che il cristianesi- 
mo non poteva essere integrato nel mondo romano allo stesso modo delle religioni naturali. Si fonda su un ideale di trascendenza. Il problema era che, inizialmente, la ragione e la fede non sono compatibili. Il cristianesimo è venuto come un sistema di pensiero, ma, al contrario della filosofia, non è stato inventato dalla ragione. La filosofia quindi, era in qualche modo scandalizzata dal cristianesimo, che era una verità coerente, ma non giustificata da niente. Le verità del cristianesimo (p. es. Il Dio Creatore, la Santa Trinità, l'Incarnazione, la Verginità di Maria) non hanno alcuna possibilità di essere dimostrate. Il cristiano, rispondendo alle perplessità dei filosofi, non poteva giustificare le verità in cui crede, ma poteva elaborare un sistema di pensiero che le spieghi, che cerchi di renderle più chiare: ed è questo quello che hanno fatto i Padri della Chiesa. Loro hanno tentato di illustrare razionalmente ciò che caratterizza un dato soprarazionale, dato di fede.

Diverse, dunque, sono state le forme con le quali i Padri d'Oriente e d'Occidente sono entrati in rapporto con le scuole filosofiche. [...] Riuscirono, infatti, a far emergere in pienezza quanto risultava ancora implicito e propedeutico nel pensiero dei grandi filosofi antichi. Costoro avevano avuto il compito di mostrare in quale modo la ragione, liberata dai vincoli esterni, potesse uscire dal vicolo cieco dei miti, per aprirsi in modo più adeguato alla trascendenza. Una ragione purificata e retta, quindi, era in grado di elevarsi ai livelli più alti della riflessione, dando fondamento solido alla percezione dell'essere, del trascendente e dell'assoluto. Proprio qui si inserisce la novità operata dai Padri. Essi accolsero in pieno la ragione aperta all'assoluto e in essa innestarono la ricchezza proveniente dalla Rivelazione (Giovanni Paolo II 1998, 41).

Quest'eredità del pensiero cristiano, concepito come l'incontro tra fede e ragione, è stata estremamente espressa nella filosofia e teologia scolastica da Tommaso d'Aquino.

\section{L'obiezione evidenzialista contro la fede}

Da quanto è stato detto sino ad ora, risulta che esiste nella nostra cultura "la crisi del senso" che deriva spesso dalla frammentarietà del sapere, che 
ha spesso un carattere scientifico-particolare, e di conseguenza la verità è concepita come pura evidenza scientifica; ma dall'altra parte esiste nel mondo il cristianesimo, che evidentemente è venuto come un sistema di pensiero, ma, al contrario della filosofia, non è stato inventato dalla ragione: le verità cristiane come coerenti, ma senza la possibilità di essere dimostrate. Quindi spontaneamente nasce l'obiezione di fronte alla fede cristiana: "L’oggetto della fede non è evidente, allora perché voi cristiani dite che l'oggetto della fede sia vero?”. Questo punto di partenza per la nostra riflessione si chiama l'obiezione evidenzialista contro la credenza (belief). Sull'importanza e sulla necessità delle credenze aveva riflettuto con grande efficacia Sant'Agostino ma anche più recentemente J.H. Newman. Loro hanno considerato la credenza come un qualsiasi contenuto cognitivo che si può caratterizzare come "pigro e passivo", perché accogliamo e facciamo nostre le credenze per una serie di motivi dei quali non siamo consapevoli, e questi non distruggono la nostra struttura noetica. Alvin Plantinga, ben noto a livello internazionale come filosofo della religione e di teologia naturale, sostiene che sia la credenza teistica che quella cristiana possono affermarsi "basilarmente", cioè senza il supporto di argomenti o dimostrazioni. Lui spiega che per evidenzialismo nel pensiero filosofico-religioso si intende la posizione secondo cui non è possibile sostenere che Dio esiste, senza portare a sostegno di questa tesi adeguate argomentazioni o prove. Ma come giustamente nota Plantinga, il problema cruciale è perché "l'obiettore ritiene vi sia un obbligo prima facie di cercare di non credere in Dio in mancanza di evidenza?" (Plantinga 2011, 36). In altre parole, secondo l'obiettore l'ateismo è obbligatorio in assenza di una evidenza, ossia di una prova a sostegno dell'esistenza di Dio. "Il dibattito non può iniziare dall'assunzione secondo cui Dio esiste” (Plantinga 2011, 22), allora come credente "io ho il dovere prima facie di ottemperare a quest'ordine: avere evidenza o non credere" (Plantinga 2011, 31). Quindi, questo tipo di affermazioni esprime una certa normatività: il credente è obbligato a non credere. Ma perché deve essere così? Si può obiettare che "naturalmente è anche vero che il dibattito non può iniziare neanche dall'assunzione secondo cui Dio non esiste” (Plantinga 2011, 22). Non ci sono le eccezioni 
per questa normatività? Secondo Plantinga ci sono circostanze nelle quali, anche se si è in assenza di prove, sarebbe razionalmente accettabile sostenere tali convinzioni senza l'obbligo di abbandonarle. L'autore americano sottolinea (con la concordanza con il notevole riscontro nella tradizione classica, secondo cui un'azione va giudicata in base all'oggetto, alle circostanze e al fine), che in una diversa circostanza le nostre convinzioni intellettuali possono cambiare. Insomma, fino a quando mancano motivi per abbandonare le proprie convinzioni, queste possono essere affermate in maniera basilare - senza evidenza e dimostrazioni. Quindi, non vi sono ragioni per escludere l'affermazione che Dio esiste dalle proposizioni basilari. L'evidenzialismo riguardante la credenza religiosa non cade nell'auto-contraddizione, perché non si deve dimostrare "ogni proposizione” (quindi anche esso stesso) dentro il sistema del pensiero. Invece, il fondazionalismo oltre le proposizioni da dimostrare, riguarda anche il rapporto tra proposizioni basilari ("primi principi”) e proposizioni da esse derivate. Plantinga spiega quali siano i criteri usati per il fondazionalismo per conseguire la definizione di basilare. Afferma che i criteri sono: 1. l'auto-evidenza - in una proposizione il soggetto implica il predicato, 2. l'evidenza sensibile al principio del sapere si pongono i sensi, 3. l'incorreggibilità - riduce ogni certezza al contenuto del pensiero. Come Plantinga fa la critica del fondazionalismo? Primo, lui mostra che ci siano anche altre forme di basilarità, non solo queste sopra menzionate. Secondo, dato che la basilarità non si riduce solo alle forme sopra elencate, la credenza in Dio non potrebbe essere considerata come l'affermazione basilare? Terzo, Plantinga mostra che sia contraddittorio accettare l'affermazione "solo le affermazioni sopra elencate sono basilari”, perché essa stessa non è né auto-evidente, né evidente dai sensi, né incorreggibile. Si è già più volte sottolineato che colui che afferma che Dio esiste, anche se non è capace di argomentare efficacemente a sostegno della propria tesi, ha pieni diritti dal punto di vista epistemologico a suo sostegno. Come ha precisato Plantinga ogni uomo può credere senza soccombere dinanzi ad accuse di irrazionalità o di deprivazione intellettuale (Plantinga 2011, 9-36; Di Ceglie 2011, VII-XX; Di Ceglie 2014, 179-193). 


\section{La caratteristica della fede e della ragione}

Dopo aver rilevato che la credenza teistica non è irrazionale, ora ci si può domandare qual'è la specificità della fede cristiana nel contesto del rapporto con la ragione. Tommaso d'Aquino scrive che:

Infatti la scienza di una cosa non è compatibile con l'opinione, poiché la scienza esige che chi conosce veda che l'oggetto conosciuto non può essere diversamente, mentre l'opinione implica l'accettazione di questa possibilità. Se invece si tiene una cosa per fede si deve affermare l'impossibilità che essa sia diversamente, data la certezza della fede; tuttavia una stessa cosa non può essere sotto lo stesso aspetto oggetto di scienza e di fede, poiché ciò che è conosciuto per scienza è visto, mentre ciò che è creduto non è visto, come si è spiegato [nel corpo] (ST, II-II, q. 1, a. 5, ad 4).

In questo testo Tommaso nota che la fede si assimilia alla scientia ma anche all'opinione. Come dice l'Aquinate, scientia è la conoscenza prodotta per inferenza da quanto è visto come vero: "Qualsiasi scienza dipende da alcuni princìpi per sé noti, e quindi evidenti. Quindi tutto ciò che è oggetto di scienza in qualche modo è oggetto di visione" (ST, II-II, q. 1, a. 5). Quindi, Tommaso, seguendo Aristotele, sostiene che la scientia è un insieme delle evidenze sensibili e di proposizioni sillogisticamente dedotte da primi principi auto-evidenti. Quest'utlimi sono conosciuti immediatamente per intuizione (l'intelletto). Ma Tommaso in modo molto chiaro distingue le conoscenze auto-evidenti colte attraverso se stesse (per se nota) da quelle colte tramite qualcos'altro (per aliud nota) (ST, I-II, q. 57, a. 2). Dunque l'immagine della conoscenza scientifica in Tommaso è così: "conosciamo ciò che è auto-evidente e ciò che si inferisce da quanto è auto-evidente per il tramite di argomentazioni auto-evidenti. La conoscenza consiste nella scientia e nell'intellectus. Intuendo cogliamo i principi primi, verità auto-evidenti; da questi poi inferiamo o deduciamo ulteriori verità" (Plantinga 2011, 41). Quindi, secondo Tommaso:

Il sapere che si caratterizza per evidenza razionale è quello il cui oggetto è conosciuto a motivo di una piena manifestazione con cui si offre al soggetto del 
conoscere. Nel passo appena menzionato [ST, II-II, q. 4, a. 1], Tommaso spiega che l'oggetto del sapere, quando è evidente, muove intelletto all'assenso, direttamente (è il caso dell'intuizione) o indirettamente (è il caso della mediazione del ragionamento) (Di Ceglie 2014, 24).

D'altra parte, nel sopra menzionato testo di Tommaso, l'opinione implica l'accettazione di possibilità che l'oggetto può essere conosciuto diversamente: non c'è certezza riguardo all'oggetto. Qual'è la posizione della fede in questa considerazione? Possiamo considerare che la fede si colloca a metà strada fra scientia e opinio. Perché? Da una parte, la fede gode di massima certezza. Dall'altra parte, la condivide la non evidenza razionale con l'opinione (Di Ceglie 2014, 39-40). Ma si deve procedere per gradi, e spiegare innanzitutto che cosa è la fede secondo Tommaso.

L’Aquinate sa che la parola "fede" può esprimere diverse realtà, come si è già detto menzionando le considerazioni di Sant'Agostino e J.H. Newman sulla credenza. Tommaso sottolinea che per "fede" si intendano tutte le forme di sapere il cui oggetto risulti inevidente (fides communiter sumpta). Ma nel caso della fede, in generale, l'oggetto è inevidente e dunque l'intelletto può essere spinto ad assentirvi "non perché è mosso adeguatamente dal proprio oggetto, ma per una scelta volontaria, che inclina più verso una parte che verso l'altra" (ST, II-II, q. 1, a. 4). Ad enfatizzare ulteriormente, Tommaso vuole dire che in mancanza di evidenza dell'oggetto, l'assenso proprio di ogni fede si verifica per un moto della volontà. Per questo l'Aquinate prende la definizione di fede da Agostino, secondo cui "la fede è pensare con assenso; colui che crede, pensa; e credendo pensa, e pensando crede" (Agostino, De praedestinatione sanctorum, 2, 5). Da quanto è stato detto sinora, Tommaso porta l'attenzione sul possesso della certezza (convictio) assieme all'inevidenza dell'oggetto:

L'intelletto di chi crede viene determinato a una data cosa non dalla ragione, ma dalla volontà. Quindi l'assenso è qui preso come un atto dell'intelletto in quanto determinato dalla volontà (ST, II-II, q. 2, a. 1, ad 3).

[...] poiché l'intelletto del credente viene convinto dall'autorità di Dio ad accettare le cose che non vede (ST II-II, q. 4, a. 1). 
Insomma, la definizione della fede secondo Tommaso è: "atto dell'intelletto che dà l'assenso alla verità divina sotto la spinta della volontà mossa da Dio per grazia” (ST, II-II, q. 2, a. 9). Quindi Tommaso ritiene che la causa propria e principale della fede è Dio "che muove l'uomo interiormente ad accettare le verità di fede" (ST II-II, q. 6, a. 1). Si crede a causa dell'autorità di Dio che si rivela a chi non può ingannare né essere ingannato. Ovviamente qui è affermata la posizione del credente che ritiene che la fede sia causata da Dio. "Di conseguenza, poichè la fede è causata da Dio essa risulta in assoluto più certa" (Di Ceglie 2014, 52). Ma l'adesione dell'uomo alle verità di fede è dovuta anche a un fattore esteriore: "la constatazione dei miracoli o l'esortazione di chi induce alla fede” (ST II-II, q. 6, a. 1). È bene rilevare che come si è già detto, ci sono i tre usi della ragione che secondo Tommaso sono possibili in materia di fede (Di Ceglie 2014, 42-44). Conseguimento dei preambula fidei. Ci sono presupposti logici del dato rivelato che sono previ, ma la fede non dipende dalla possibilità di raggiungere loro (ST, II-II, q. 1, a. 5, ad 3). La coerenza interna della fede, è che cioè i credenti hanno la conoscenza - notitiam habent - del fatto che l'oggetto della fede è da credersi. In oltre parole, ci sono le ragioni di fede, ma non nel senso dimostrativo, ma nel senso persuasivo, "in quanto mostrano che non è impossibile quanto la fede propone” (ST, II-II, q. 1, a. 5, ad 2). In questo punto si possono includere le suddette constatazioni dei miracoli, ma anche il ruolo del testimone della fede, perché la fede è la conoscenza acquisita mediante la fede negli altri che sono degni di credere (Livi 2007, 27-52). Nel terzo uso, mentre la fede in sè rimane inevidente per la definizione, la filosofia può difendere la fede dalle affermazioni che le si oppongono, e in realtà se la fede è vera, le obiezioni ad essa devono essere false. In conclusione, “la ricerca dell'evidenza razionale (relativa ai motivi e non all'oggetto della fede) agisce solo al livello del movente esteriore, non rispetto a quello interiore" (Di Ceglie 2014, 47; Fabro 2007, 45-48).

E` bene rilevare che Tommaso sostiene che "nel sapere si possono considerare due cose: l'adesione di colui che sa alle verità scientifiche e la considerazione attuale di tali verità" (ST, II-II, q. 2, a. 9, ad 2). Quindi non è vero che laddove si goda di evidenza non è necessaria la cooperazione 
della volontà. Secondo Tommaso anche nel campo del sapere si deve dare lo spazio per la dimensione affettiva e della volontà: considerazione attuale - consideratio rei scitae. Da un lato esiste l'assensus, ma dall'altro ci sono le situazioni particolari, dove si manifesta il desiderio e la tensione a sviluppare questo o quell'aspetto della riflessione razionale. Quindi, la libertà agisce anche nel campo delle conoscenze scientifiche. Come si è già detto, la differenza fra la scienza e la fede consiste nel fatto che nell'ultima l'intelletto agisce interamente sotto la forza della volontà.

Si è già più volte sottolineato che nel pensiero di Tommaso l'atto di fede va considerato in una triplice dimensione: l'intelletto, la volontà, gli affetti e relazione con Dio. Si è evidenziato che Tommaso distingue la fede dall'opinione, dalla supposizione e dalla scienza. Si passa ora a considerare la tripartizione tommasiana riferita all'oggetto della fede (ST, II-II, q. 2, a. 2). L'Aquinate distingue fra "credere Dio" (credere Deum), "credere a Dio" (credere Deo), "credere in Dio" (credere in Deum). Come ben nota Di Ceglie (2014, 93):

i primi due aspetti definiscono l'ambito di esercizio dell'intelletto, e più precisamente il contenuto della fede (l'oggetto materiale: credere Deum) e il modo in cui esso viene proposto al fedele (l'oggetto formale: credere Deo); il terzo aspetto individua invece il compito proprio della volontà, la quale, poiché mira e tende al bene, spinge l'intelletto a dare l'assenso alla verità rivelata.

La fede, come molte volte si presenta la posizione tommasiana, non può essere ridotta solo all'opera dell'intelletto. "L'atto della fede dice ordine sia all'oggetto della volontà, che è il bene e il fine, sia all'oggetto dell'intelletto, che è il vero" (ST, II-II, q. 4, a. 1). In altre parole, nel caso della fede l'oggetto della conoscenza è anche la fonte di essa. Il fedele tende a Dio perché è mosso interiormente verso di lui, ma tende a Dio perché è Dio che si rivela. Evidentemente "credere in" si può sostituire anche con la parola "confidare" (Di Ceglie 2014, 94-95). Di conseguenza secondo Di Ceglie credere in Deum è il nucleo della dottrina tommasiana della fede: "che Dio sia non solo l'oggetto e il fine ma anche il principio e l'origine della fede, non solo perché rivela determinate verità, ma anche e soprattutto perché, pur salvaguardandone la libera scelta da parte dell'uomo, muove i credenti ad accoglierle" (Di Ceglie 2014, 95). 
Ben si vede questa posizione di Tommaso quando lui considera la carità come la virtù che perfeziona la fede (ST, II-II, q. 4, a. 3). Perché la carità è la forma della fede? Perché "ordina gli atti di tutte le altre virtù al fine ultimo, per cui in tal modo dà la forma agli atti di tutte la altre virtù" (ST, II-II, q. 23, a. 8). Più precisamente "si dice che la carità è la forma delle altre virtù non in maniera essenziale o esemplare, ma piuttosto in maniera efficiente" (ST, II-II, q. 23, a. 8, ad 1). Il ruolo decisivo che la carità svolge rispetto alla fede emerge anche quando Tommaso riflette sui sopra menzionati preambula fidei. "Quando invece uno ha la volontà da credere le verità di fede per la sola autorità di Dio, anche se scopre delle ragioni per dimostrare qualcuna di queste verità, p. es. l'esistenza di Dio, non per questo vede eliminato o sminuito il merito della fede" (ST, II-II, q. 2, a. 10, ad 1), perché "le ragioni dimostrative a favore di verità di fede previe però ai vari articoli, sebbene diminuiscono la formalità della fede in quanto rendono evidenti le cose presupposte, tuttavia non sminuiscono quella della carità, che rende il volere pronto a credere anche le cose inevidenti” (ST, II-II, q. 2, a. 10, ad 2). Anche Tommaso sottolinea che anche l'amore privo di contenuto intellettuale è dono di Dio, cioè tensione e amore per lui. Si può riassumere che il desiderio di conoscere sia proporzionale alla carità che spinge verso Dio e verso le sue opere. Ma anche quando manca il contenuto intellettuale, rimane l'animo disposto a credere (ST, II-II, q. 2, a. 5). Come si è già detto, la definizione di fede di Agostino e Tommaso dimostrano chiaramente che la fede "si caratterizza dunque per un'inquietudine e un desiderio di affermare sempre più la verità creduta che non cessa mai di animare l'intelligenza del cristiano" (Di Ceglie 2014, 50). Si può dire che nel campo della fede: chi non ama, non può conoscere. Insomma, il "desiderio di credere", "l'amore per la verità creduta”, costituiscono il nucleo dell'epistemologia teologica di Tommaso (Di Ceglie 2014, 96-102).

\section{Le possibili comprensioni del rapporto tra fede e ragione in Tommaso}

Si passa ora a offrire una sintesi della relazione tra fede e ragione espressa nella formula: primato della fede e autonomia della ragione (Di Ceglie 
2015). Si è già più volte sottolineato, che l'Aquinate è consapevole delle diverse esigenze della fede e della ragione ma anche sembra che quella formula riassumi tutto quanto sostenuto finora.

Secondo Di Ceglie, da una parte, ci sono molte interpretazioni che sottolineano che la teologia di Tommaso costituisce il risultato degli sforzi della sola conoscenza naturale, senza alcun influsso della fede. Uno dei studiosi che rappresenta questa tradizione è p.es. Ralph McInerny (2006). Lui fa riferimento a diverse affermazioni tommasiane che dimostrano sopra menzionata tesi. Ad esempio, Tommaso al principio della Summa theologiae scrive: "L'esistenza di Dio, come pure altre affermazioni che possono avanzarsi rispetto a Dio per mezzo della ragione naturale - come si dice in Rm 1,19 - non sono articoli di fede ma preamboli agli articoli; infatti la fede presuppone la conoscenza naturale, come la grazia presuppone la natura e la perfezione presuppone il perfettibile” (ST, I, q. 2, a. 2, ad 1, cit. in McInerny 2006, 29). Questo si presenta come l'impostazione della sola conoscenza naturale. McInerny poi fa riferimento a un altro testo: "Della stessa cosa secondo lo stesso aspetto nel medesimo tempo e presso la stessa persona non possono darsi né scienza e opinione né scienza e fede, sebbene per diversi motivi. [...] ... la medesima cosa secondo il medesimo aspetto non può essere contemporaneamente conosciuta e creduta, poiché ciò che è conosciuto è visto, mentre ciò che è creduto non è visto" (ST, II-II, q. 1, a. 5 , ad 4). In questo passo, l'Aquinate sostiene la posizione dell'incompatibilità di fede e scientia. Per lui, nella sua dottrina dell'evidenza, l'ultima è la qualità delle cose "che da sé stesse muovono l'intelletto o i sensi alla loro conoscenza", mentre, come si è già detto, l'oggetto della fede non è evidente e per questo motivo l'assenso dell'intelletto è dato all'oggetto della fede "per il comando della volontà mossa da Dio per grazia”. Da questo passo si può desumere che, laddove gli oggetti del sapere sono evidenti, l'intelletto li conosca senza l'influsso della volontà mossa dalla grazia. Si è già notata una cosa simile con il caso dei preambula fidei. Tommaso sostiene che "nulla vieta che ciò che in sé è dimostrabile e oggetto di scienza sia accolto nella fede da chi non ne capisce la dimostrazione" (ST, I, q. 2, a. 2, ad 1), ma dall'altra parte scrive che è necessario che la verità dimostrabili 
"siano presupposte almeno per fede da coloro che non ne colgono la dimostrazione” (ST, II-II, q. 1, a. 5, ad 3). Quindi se l'oggetto del sapere sia passibile di dimostrazione, la ragione risulti preferibile a fede. McInerny conclude che nella prospettiva tommasiana la teologia naturale può essere sviluppata da chi crede e da chi non crede. Invece, secondo lui, il pensiero di Étienne Gilson "finì per confinare la filosofia di Tommaso al contesto teologico tanto che risulta difficile capire come la filosofia così intesa possa essere condivisa anche dai non credenti" (McInerny 2006, 159). Anche in altro luogo si può trovare il testo tommasiano per sostenere la posizione secondo cui la ragione naturale lavora senza alcun influsso da parte della fede: “Un'opinione falsa è come un difetto dell'intelletto [...] Ora, il difetto è qualcosa di accidentale poiché non rientra nell'intenzione della natura, e ciò che è accidentale non può essere sempre e in tutti [...]. Quindi il giudizio che tutti danno della verità non può essere falso" (CG, II, 34). Come spiega Tommaso, se "il giudizio che tutti danno della verità non può essere falso", quindi è affidabile punto di partenza di ogni dimostrazione. Questa spiegazione, che emerge da posizione puramente razionale, si basa sulla necessità di fondare le dimostrazioni su principi da tutti condivisi, ed è costituita da un argomento da tutti condivisibile. In questo ambito la fede non sembra svolgere alcun ruolo.

Secondo Di Ceglie, l'altra prospettiva che riguarda il rapporto tra fede e ragione in Tommaso ritiene che la fede cristiana abbia determinato l'intentio profundior dell'indagine razionale di Tommaso, almeno nel caso delle verità che, oltre ad essere conseguibili per sola ragione, sono state anche rivelate. Le parole di Étienne Gilson non lasciano dubbi: “Tommaso sarebbe stato molto sorpreso di sentirsi dire che, nel dare dimostrazione filosofica di quelle verità che sono state rivelate da Dio agli uomini e che sono filosoficamente dimostrabili, egli, sebbene commentasse la parola di Dio, stava dando libero corso a un'attività non teologica” (Gilson 2006, 321). Secondo il filosofo francese l'interpretazione del pensiero tommasiano, che suggerisce che non si può attribuire all'Aquinate la compresenza di ragione autonoma e di influsso della fede, è una eredità dell'età moderna che risale a Descartes (ibidem, 323). Di Ceglie nota che la posizione di Gil- 
son non è isolata. Si può trovare una lettura analoga in un altro interprete dell'Aquinate, John Jenkins (Di Ceglie 2015, 4-5). Ad enfatizzare ulteriormente il supporto che la sopra menzionata posizione trova nel pensiero di Tommaso, si può fare riferimento a un testo: "Se nelle sentenze dei filosofi si trova qualcosa che risulta contrario alla fede, non si tratta di filosofia ma piuttosto di abuso della filosofia dovuto al fatto che la ragione non è stata esercitata adeguatamente. E perciò è possibile trovare l'errore in questione a partire dai principi della filosofia, mostrando che si tratta di qualcosa di impossibile o di non necessario" (Tommaso d'Aquino 1997, q. 2, a.3). Sembra che Tommaso sostenga che si possono rifiutare le conclusioni di un ragionamento in caso di contrasto con la verità di fede. Ma forse qualcosa di simile andrebbe sostenuto anche nel caso in cui non fosse il conflitto sopra menzionato. In altre parole, la ragione potrebbe essere in errore anche quando secondo l'argomentazione tutto portasse a sostenere che le conclusioni di un ragionamento possano risultare veramente convincenti. Quindi, si può ipotizzare che per Tommaso la certezza (assenza di dubbio) non è offerta dagli argomenti razionali ma dalla fede: "Si è molto più certi di ciò che solo si ascolta da Dio, il quale non può sbagliarsi, che di ciò che si capisce con la propria ragione, la quale può sbagliare” (ST, II-II, q. 4, a. 8, ad 2).

Da quanto è stato detto sinora, le due posizioni sopra presentate appaiono chiaramente alternative ma anche opposte l'una all'altra. Come si è già detto una nega che l'Aquinate svolga la riflessione filosofica sotto l'influenza della fede, l'altra invece afferma una simile influenza, e ritiene che la fede cristiana abbia determinato l'intentio profundior dell'indagine razionale di Tommaso, almeno nel caso delle verità che, oltre ad essere conseguibili per sola ragione, sono state anche rivelate. E` molto significativo che entrambe le posizioni trovano il supporto nei testi di Tommaso. Secondo Di Ceglie l'opposizione può essere superata se venisse considerata nella sua interezza la plurivoca concezione tommasiana della fede e conseguentemente del suo rapporto con la ragione. Da un lato per fede si intende l'adesione personale a Cristo. Quest'adesione è motivata principalmente dall'amore di Dio (la grazia divina) del quale il cristiano partecipa e non si 
può semplicemente negare che questa relazione non intervenga in qualche modo nella vita intellettuale del cristiano. Dall'altro lato per fede si intendono i contenuti noetici (un tipo di sapere intorno al messaggio cristiano). Ma questo tipo del sapere non può prendere parte all'investigazione filosofica, perché c'è la mancanza di evidenza razionale. Questi vari aspetti della fede si trovano nella ben nota distinzione, che risale a Sant'Agostino: da una parte fides qua (indica l'atto stesso con il quale il credente, sotto l'azione della grazia, si affida a Dio che si rivela e ne assume il contenuto come vero) e fides quae (indica il contenuto della fede che viene accolto; le diverse verità di fede che sono accolte e credute come un tutt'uno, in un solo atto). Si è già più volte sottolineato, che per Tommaso la fede è “atto dell'intelletto che dà l'assenso alla verità divina sotto la spinta della volontà mossa da Dio per grazia” (ST, II-II, q. 2, a. 9). Ma la fede consiste non solo nell'opera dell'intelletto, anche in quella della volontà, ed è dovuta all'intervento e al sostegno offerto da Dio. Questa constatazione corrisponde con sopra menzionata un'analoga tripartizione tommasiana riferita all'oggetto della fede: "credere Dio" (credere Deum), "credere a Dio" (credere Deo), "credere in Dio" (credere in Deum) (ST, II-II, q. 2, a. 2). Come ben nota Di Ceglie (2015, 7), solo la prima delle tre dimensioni dell'atto di credere, credere Deum, definisce il carattere intellettuale della fede, cioè un atto dell'intelletto con relativi contenuti noetici (fides quae). Mentre la fides qua è indicata dagli altri due aspetti: credere Deo significa che l'atto di fede dipende da Dio, cioè la sua rivelazione che si dà l'assenso nella fede; credere in Deum - l'oggetto in questione è considerato qui come il fine ultimo, il bene, ma non un bene come un altro, ma come bene in sé, a cui tende la volontà.

Dunque, si ben vede, che secondo la spiegazione tommasiana la fede non è riducibile alla sola opera dell'intelletto, perché l'Angelico riconosce il ruolo decisivo della volontà e da elementi affettivi. Inoltre, per Tommaso la fede non è riducibile alla sola opera dell'uomo. Infatti, l'oggetto della fede (credere Deum) è anche l'origine della conoscenza. Ma lo è exterius, perché eccede le capacità della ragione umana, e per questo l'uomo non risulta capace di conoscerlo se non perché è Dio che si rivela (credere Deo). Ma 
dall'altra parte lo è anche interius, poiché l'assenso ad quelle verità che eccedano le capacità umane deve provenire "da un principio sovrannaturale che muova interiormente, ossia da Dio" (ST, II-II, q. 6, a. 1). Quest'ultimo aspetto sarebbe credere in Deum, cioè la tensione della volontà al Bene. La rivelazione è voluta da Dio (exterius), e quindi lo si ama, grazie all'opera che lui compie nei fedeli (interius) quando li muove all'assenso. Ovviamente, per Tommaso ci sono diversi ragioni, che permettono all'intelletto di dare l'assenso alla verità creduta, ma che veramente muove gli uomini verso di lui è Dio - la causa propria e principale della fede (ST II-II, q. 6, a. 1, ad 1). Secondo Di Ceglie, come si è già detto, si può dire che credere in Deum è il nucleo della dottrina tommasiana della fede, che anche in maniera sufficiente rappresenta la grande tradizione del pensiero cristiano: che Dio sia non solo l'oggetto e il fine della fede, ma soprattutto il principio e l'origine dell'essa. Infatti, Tommaso spiega che "la carità si dice forma della fede in quanto per mezzo di essa l'atto di fede è reso perfetto" (ST, II-II, q. 4, a. 3). L'amore di Dio e per Dio costituisce il cuore della fede cristiana e il motivo dell'esigenza di conoscere Dio. E` bene rilevare, che secondo Tommaso la conoscenza di Dio dipende dall'amore che si nutre nei suoi confronti:

L'intelletto, più partecipa della luce della gloria, più perfettamente vede Dio. Ma partecipa di più della luce della gloria chi ha un grado superiore di carità, poiché là vi è maggiore carità dove vi è maggiore desiderio, quel desiderio che in qualche modo rende colui che desidera adatto e pronto a ricevere la cosa desiderata. Da cui deriva che colui che avrà avuto più carità, vedrà più perfettamente Dio (ST, I, q. 12, a. 6).

Ogni opera del cristiano, anche quella filosofica, dovrebbe nascere dall'amore per Dio e come sua piena espressione. Nel testo dalla Summa contra Gentiles Tommaso dice che:

Il filosofo e il credente considerano aspetti diversi delle cose. Il filosofo infatti prende in esame ciò che ad esse conviene secondo la loro propria natura, come ad esempio il salire verso l'alto da parte del fuoco. Il credente invece considera solo ciò che conviene alle cose secondo la loro relazione a Dio, come il fatto che sono state create da Dio, che sono soggette a lui, e così via (CG, ii, 4). 
Secondo lui, il fidelis e il philosophus indagano lo stesso oggetto (la realtà) con la medesima scientificità, ma è diverso l'interesse che li muove. E` molto significativo che l'Aquinate conclude questa riflessione dicendo che "dopo aver trattato di Dio nel primo libro, rimane ora da occuparsi di tutto ciò che da lui procede" (ibidem). Allora lui sostiene che sta svolgendo l'opera del fidelis. Insomma, per Tommaso l'amore verso Dio è la causa dell'obbedienza alla sua rivelazione e del desiderio di comprendere ogni cosa a partire da essa.

Si è già detto più volte, che per Tommaso la fede è "atto dell'intelletto che dà l'assenso alla verità divina sotto la spinta della volontà mossa da Dio per grazia”. E si è anche già sottolineato, che credere in Deum è il nucleo della dottrina tommasiana della fede: la fede non sole come un certo sapere ma anche come il desiderio di riflettere ulteriormente su un sapere al fine di ampliarlo sempre più. Ora si deve interrogarsi con maggiore precisione, come spiega Di Ceglie, circa tre cose: 1 . se la carità rende perfetta la fede del credente - "l'amore per la verità creduta" come Tommaso la chiama non può essere disfarsene dove lui deve compiere un'indagine con la ragione naturale; 2 . come Tommaso risulta capace di garantire l'autonomia della ragione dalla fede, perché la ragione cerca l'evidenza ma di quest'ultima la fede manca per definizione; 3. come è possibile che, mentre "l'amore per la verità creduta" non è abbandonata nel percorso svolto dalla ragione naturale, la ragione può funzionare autonomamente.

Tommaso, quando riflette sul modo di argomentare a proposito delle verità di fede, scrive così:

Le ragioni umane portate a favore della fede possono avere due diversi rapporti con la volontà del credente. Possono essere antecedenti, come quando non si avrebbe la volontà di credere, o non la si avrebbe pronta, se non si fosse indotti a ciò da una ragione umana. $\mathrm{E}$ così le ragioni umane addotte diminuiscono il merito della fede [...]. In un altro modo, le ragioni umane possono essere conseguenti rispetto alla volontà di chi crede. Un uomo che ha la volontà pronta a credere ama la verità creduta, vi riflette e abbraccia le ragioni umane a supporto, se può trovarne. $\mathrm{E}$ in questo caso le ragioni umane non escludono il merito della fede, ma sono il segno di un merito più grande (ST, II-II, q. 2, a. 10). 
L'Aquinate sottolinea qui il valore intellettuale “dell'amore per la verità creduta", cioè l'amore di Dio e per Dio che fa tendere a Dio e riflette su di lui e su ogni cosa a partire da lui e in accordo con lui. Inoltre, Tommaso spiega, che la disposizione a credere ("volontà pronta a credere") opera anche quando l'oggetto dell'argomentazione sia evidente. In questo caso la fede come complesso di verità inevidenti viene meno, mentre la forma della fede - la carità che conferisce perfezione e pienezza alla fede - persiste. E` il caso dei preambula fidei: "Le dimostrazioni portate a sostegno delle verità della fede, di quelle previe agli articoli, sebbene diminuiscano la misura della fede in quanto rendono evidenti gli oggetti da credere, non diminuiscono però quella della carità, grazie alla quale la volontà è pronta a credere quegli oggetti anche se non risultano evidenti” (ST, II-II, q. 2, a. 10, ad 2). Evidentemente per l'Aquinate "la misura della fede" risulta diminuita o annullata, perché l'evidenza è raggiunta. I preambula fidei divengono evidenti. Ma l'amore di Dio e per Dio ("la misura della carità") rimane operativa. Da questo consegue, che per il credente è possibile conoscere e credere allo stesso tempo. Questo potrebbe contraddire il sopra menzionato passo da ST, II-II, q. 1, a. 5, ad 4 (p. 10). Ma come spiega Di Ceglie, per Tommaso non può essere sostituita la grazia divina con la sua dignità soprannaturale dall'opera umana: "la grazia è più perfetta dalla natura, per cui non viene meno laddove l'uomo può ricevere perfezione secondo natura” (ST, II-II, q. 9, a. 1). In altre parole, il conoscere, come conseguimento dell'evidenza fondato sulla ragione naturale, non sostituisce il credere, come adesione alla rivelazione motivata dall'amore di Dio e grazia divina.

Si passi ora a considerare come Tommaso risulta capace di garantire, che nel caso della fede la ragione può funzionare autonomamente. Come il credente, Tommaso sostiene, che la causa della fede è più certa, perché essendo Dio e non la ragione umana. Ma dal punto di vista del soggetto della fede (cioè il credente) "si dice più certo ciò che l'intelletto umano consegue con maggior completezza" (ST, II-II, q. 4, a. 8) e che "la perfezione dell'intelletto e della scienza supera la conoscenza di fede per una maggiore chiarezza" (ST, II-II, q. 4, a. 8, ad 3). Mentre la ragione cerca l'evidenza, la fede, intesa come assenso ad affermazioni non-evidenti (credere Deum), 
non tende a questo scopo. La certezza della fede è determinata dalla grazia divina, non dall'evidenza razionale. Quindi dal punto di vista del soggetto, la ragione umana risulta in grado di conseguire la certezza senza l'influsso della fede. Secondo Di Ceglie si può facilmente vedere sopra menzionata prospettiva - la relazione tra fede, ragione e carità - nel passo nel quale Tommaso discute la possibilità che le conclusioni della ragione neghino le verità della fede: "Poiché la fede poggia sulla verità indefettibile, e poiché è impossibile dimostrare ciò che è contrario al vero, risulta evidente che le prove addotte contro la fede non sono dimostrazioni ma argomenti da riconsiderare" (ST, I, q. 1, a. 8). Tommaso riconosce, che è la sola ragione ad aver il compito di conseguire l'evidenza. Perché dopo aver rifiutato gli argomenti razionali che contraddicono con la fede, la ragione ha il compito di riprendere il cammino a partire dai suoi principi. Quindi, l'Aquinate esprime una notevole fiducia nei confronti della potenzialità della ragione. Perché Tommaso richiede una nuova investigazione condotta dalla sola ragione? Da una parte c'è la sopra menzionata fiducia nella ragione, ma dall'altra parte la certezza della fede non dipende dalla ragione naturale, come si è già detto. Infatti, essa consiste nell'adesione alle verità rivelate. Quindi la certezza della fede è la certezza dell'amore che per grazia divina lega i credenti a Dio e li rende pronti ad accogliere come vero quanto egli ha rivelato. In virtù di una simile certezza, i credenti possono respingere qualsiasi argomento, anche molto convincente, per il solo suo essere in contrasto con le verità della fede. In conseguenza, l'amore che i credenti nutrono nei confronti di Dio e la certezza della fede che riguarda la sua rivelazione è come l'orientamento della indagine razionale (la utilizza come strumento per rifiutare le obiezioni e confermare le verità rivelate) dei cristiani. Anche i cristiani assumono la verità amata e creduta come un criterio del proprio percorso speculativo. Perché sull'accordo o disaccordo con essa accettano o rifiutano le conclusioni della ragione. Di Ceglie ben nota che a questo punto possa essere elevata un'obiezione: come un criterio per l'indagine razionale dovrebbe operare solo la ragione, senza l'influsso dell'amore. Ma per i cristiani, Dio è il bene in se stesso, non un bene qualsiasi. Questo significa che quanto più ameranno lui, tanto più 
ameranno il bene e saranno buoni. Quindi l'amore per Dio non deve essere una passione che impedisce conseguire la verità. Dio è il bene in sé ma anche la verità in sé. In conseguenza, non è strana l'affermazione di Tommaso, cui scrive: "Dio è certamente ciò per cui si conoscono tutte le cose, non nel senso che le altre non si conoscano se prima non si è conosciuto lui, come capita per ciò che è auto-evidente, ma nel senso che per la sua influenza ogni cosa viene conosciuta da noi” (CG, I, II). Secondo Tommaso, grazie a Dio si può conoscere tutte le cose, perché coloro, che esercitano la ragione animati dall'amore per il bene in sé, sono spinti a rendere il cammino del conoscere. Secondo Di Ceglie queste parole di Tommaso sembrano come il riassunto della tesi sopra introdotta: una sintesi della relazione tra fede e ragione come il primato della fede e autonomia della ragione. $\mathrm{Da}$ un lato, Tommaso afferma che il discorso razionale è fondato sui principi auto-evidenti e in conseguenza le verità della fede non possono prendere parte della dimostrazione esercitata dalla ragione naturale (l'autonomia della ragione). Dall'altro lato, Tommaso afferma che grazie a Dio, "per la sua influenza ogni cosa viene conosciuta da noi”. In virtù di questa influenza divina (il primato della fede), i credenti amano Dio, nutrono fiducia in lui e credono vere le verità da lui rivelate. Insomma, le verità della fede operano come l'orientamento e criterio: il primo, perché i credenti mirano a confermare dal punto di vista razionale le verità in quale già credono; il secondo, perché nel caso della contraddizione, la ragione deve essere considerata come errante e deve riconsiderare i argomenti dall'inizio.

\section{Conclusione}

Evidentemente la posizione di Tommaso, a riguardo del rapporto tra fede e ragione, si può considerare come un tentativo di realizzare un equilibrio. "Nella sua riflessione, infatti, l'esigenza della ragione e la forza della fede hanno trovato la sintesi più alta che il pensiero abbia mai raggiunto, in quanto egli ha saputo difendere la radicale novità portata dalla Rivelazione senza mai umiliare il cammino proprio della ragione” (Giovanni Paolo II 1998, n. 78). Difatti la fede, se fosse priva di ragioni, necessariamen- 
te cadrebbe nell'irrazionalità del fideismo. Come ben mostra Tommaso, è insufficiente promuovere un atteggiamento fideistico, che suggerisce di "credere per credere". Tommaso molte volte sostiene che ci sono buone ragioni che permettono all'intelletto di dare l'assenso alla verità creduta così che la ragione deve cercarle per svolgere il suo proprio compito. D’altro canto, la fede nella concezione tommasiana non è stata ridotta a una forma di razionalismo. L'oggetto della fede - Dio stesso - è trascendente e l'umana intelligenza non può semplicemente dimostrarlo, perché c'è l'assenza dell'evidenza.

Si è già detto, che il cuore della concezione tommasiana è il terzo aspetto della fede - credere in Deum. I primi due aspetti (credere Deum, credere Deo) generalmente si riferiscono alla ricerca intellettuale. Ma il terzo aspetto consente di parlare della conoscenza che coinvolge tutta la persona umana, nei suoi aspetti intellettuali, volontari, ma anche affettivi. Da questo punto giunge la giustificazione del tema della conoscenza tramite la fede (Grabowski 2003, 190-196). A questa ipotesi può essere elevata un'obiezione che emerge quasi spontaneamente: come è possibile conoscere tramite la fede, se per definizione la fede implica l'assenza dell'evidenza? Il clima culturale nel quale viviamo riduce il significato della parola “conoscenza” solo all'aspetto che si basa sull'evidenza e i principi della logica, quindi quanto l'atto di fede in Dio sia irragionevole per l'essere umano. Si è già detto che l'obiezione evidenzialista vuole escludere $a$ priori ogni proposizione non derivata dall'evidenza empirica o dalle leggi della logica. Max Scheler diceva che quando un uomo conosce, trascende se stesso e acquista la partecipazione in una realtà conosciuta. In altre parole: conoscere significa partecipazione in una realtà. Evidentemente questo è lo scopo del percorso di Tommaso, della sua epistemologia: approfondire la definizione della conoscenza come "l'amore per la verità creduta" "il desiderio di credere”, la partecipazione nell'amore divino. Come si può spiegare questo in termini del senso comune? E` possibile credere in molte cose. Noi crediamo in molte cose perché non possiamo controllare, verificare tutto. Ma una cosa è la fede, come l'accettare qualcosa come vero - la ricerca intellettuale, e un'altra, cioè affidarsi a qualcuno - il coinvolgimento di tutta la persona 
nel relazione con l'Altro. Sia il teologo, il filosofo che il mistico conoscono Dio. I primi hanno un'esperienza intellettuale, ma i secondi hanno un'esperienza esistenziale perché per loro Dio è il fine ultimo - tutto quello che lui fa, lo fa per Dio e in Dio. Solo l'atteggiamento di affidarsi a qualcuno significa esistenziale partecipazione. E questa partecipazione, che ben spiega Tommaso, ha un nome: l'amore (la fede formata per la carità). Quindi l'Angelico sostiene che credere in Deum esprime la totalità dell'atto della fede. I mistici della Chiesa dicevano che la fede è lo scuro apprendimento di Dio tramite l'amore. Insomma, nella conoscenza tramite la fede lo scopo è come in ogni atto della conoscenza: la verità. Ma conoscere tramite la fede significa costruire la relazione con Dio nell'atto di fiducia nell'autorità di Dio, e questo atto dell'amore apre la strada per raggiungere lo scopo della ricerca umana.

\section{Bibliografia}

Di Ceglie, R. 2011. Introduzione a Dio esiste. Perchè affermarlo anche senza prove.

A. Plantinga. VII-XXIX. Soveria Mannelli: Rubbettino.

Di Ceglie, R. 2014. Credere in Deum. Ariccia: Aracne.

Di Ceglie, R. 2015. “Faith, reason, and charity in Thomas Aquinas's thought.” International Journal for Philosophy of Religion (2015). Accesso: Aprile 15, 2015. Doi: 10.1007/s11153-015-9513-6.

Fabro, C. 2007. Opere Complete. Breve Introduzione al Tomismo. Vol. 16. Roma.

Fisichella, R. 1997. Quando la fede pensa. Casale Monferrato: Piemme.

Giovanni Paolo II. 1998. Fides et ratio.

Gilson, É. 2006. “What is Christian Philosophy?” In: Gilson Reader. Selected Writings of Etienne Gilson ed. A.C. Pegis. New York 1957: Garden City. Trad it. "Che cosa è la filosofia cristiana?” In: Ragione e Incarnazione. R. Di Ceglie. Città del Vaticano 2006: Lateran University Press.

Grabowski, M. 2003. Na ścieżkach współczesności. Kraków.

Livi, A. e Silli, F. 2007. Logica della testimonianza. Quando credere è ragionevole. Roma.

Livi, A. 2008. “L'essenza razionale del cristianesimo”. Studi cattolici 52 (2008): 324-330 . 
McInerny, R. 2006. Preambula fidei. Thomism and the God of the Philosophers. Washington DC: The Catholic University of America Press.

Pangallo, M. 2004. Il creatore del mondo. Breve trattato di teologia filosofica. Roma.

Plantinga, A. 2011. Dio esiste. Perchè affermarlo anche senza prove. Trad. it. di Reason and Belief in God a cura di R. Di Ceglie. Soveria Mannelli: Rubbettino.

Ratzinger, J. “La verità cattolica.” Trad. it. Micromega 2 (2000): 41-64.

Tommaso d'Aquino. 1985. Summa Theologiae [nel testo: ST]. Edizioni Studio Domenicano. Bologna.

Tommaso d’Aquino. 1997. Super Boethium De Trinitate. A cura di C. Pandolfi, Edizioni Studio Domenicano. Bologna.

Tommaso d'Aquino. 2000. Summa Contra Gentiles [nel testo: CG]. A cura di T.S. Centi, Edizioni Studio Domenicano. Bologna. 